There are a large number of external stimuli which have more or less influence in disturbing physiologic processes and so directly affect the nervous system, which it is necessary to constantly bear in mind. The influence in the monthly rhythm in women has long been recognized, as well as the periods of fertility and menopause, and their analogues in man, adolescence, virility and senile impotence; but there are other rhythms, those of the atmosphere and temperature, spoken of above, which have a great influence on certain neurotic temperaments and on those whose biologic adjustment has been faulty.

The regular increase of crime against property in winter is only an indirect result, through the social and economic influences of temperature, but the increase of crimes of passion and indecent assaults during the months and years when the temperature is highest is the direct effect of the temperature. ${ }^{5}$ The crime of rape is most numerous in June and least so in November. ${ }^{6}$ We are all acquainted with patients whose biologio conditions are such that they offer the feeblest resistance to diurnal variations. The presence of the sun or its absence exerts a marked influence on the physical and psychic organism of certain individuals. This class of barometric individuals exhibits at night a relatively unequilibrated, emotional and restless condition associated with lessened mental inhibition. Such changing states caused the alienist of old to attribute nocturnal intensification of insane symptoms to the coincident appearance of the moon, which there is no reason to believe has ever any other influence on insane patients than that of a sensorial stimulus of a mild and customary kind (Kellogg).

A consideration of the above facts will enable one to see how dependent the habits and actions of man are upon environment and the conditions which are necessary factors in keeping this physical world in a position to support sentient beings.

We too of ten treat the nervous system as an isolated independent entity and forget that it is one of sereral, mutually dependent on each other for their common existence, which common existence is controlled to a great extent by external forces and stimuli, over which we have no guidance or rein, but to which we must ever bear a listening attitude and respectful submission.

\section{CURE OF INTERNAL HEMORRHOIDS.}

\section{BY JAMES A. GARDNER, M.D.}

$$
\text { BUFFALO, N. Y. }
$$

Undoubtedly there have been sufficient schemes advanced to cure internal hemorrhoids to rival in number the so-called "cures" of cancer. The trouble is so annoying and painful that the poor sufferer, trying each, turns from one to another and finally throws all aside as useless. The treatment of internal hemorrhoids divides itself into two classes, medical and surgical.

As regards the medical treatment, as a rule the patient has taken his druggist's advice and used all the different "cures" he has in stock, and finding no relief here he goes through the list advertised in the newspaper. He then turns to the physician. By this time the pathologic condition is such that the soothing ointment of his doctor does but little good. In the majority of cases, if the patient presented himself during the earlier stages, proper application of ich-

5 Criminal Sociology, Ferri, 1898.

6 Des attentants a la Pudeur sur les Petites flles. P. Bernard, Thése de Lyon, 1886 . thyol would reduce the inflammation and effect a cure. Most remedies contain opium and belladonna, whether advertised as such or not, and therefore give but tem. porary relief. Oftentimes, when a patient consults the physician, there is some reason why there can not be surgical interference. If the tumor protrudes, it must be replaced as soon as possible, the pain being most severe with the tumor outside of the sphincter. The congestion may be reduced by cold applications, cold sitz baths or chopped ice. Following this the tumor should be well lubricated with olive-oil and then gently pushed back in place. Occasionally it is impossible to do this and an anesthetic must be used, and once the spasm of the sphincter having been removed it is, as a rule, a simple matter to replace it. This is but temporary relief, and at the next movement of the bowels the tumor will probably return. Astringents, cold applications, with rest in the prone position, are now in order. The cold application is best made by injection; this not only contracts the blood-vessels but will also render fecal matter of such consistency that it will irritate the inflamed surface but little. This treatment does not have the extended use it should. Cold water enemas are by all means the best palliative measure.

When piles become troublesome and detrimental to health, either from their tendency to bleed or prolapse, surgical treatment is the only course to pursue in a majority of cases, as affording a complete and permanent cure. Beforeadvising an operation, make sure that the hemorrhoids are not caused by some disorder higher up, stricture of the bowel, cancer, disease of uterus or bladder, enlarged prostate or cirrhosis of liver. Hemorrhoids of pregnancy rarely need surgical interference. A number of methods have been advanced for the cure: 1. Electrolysis. 2. Nitric acid. 3. Dilatation of sphincter. 4. Crushing. 5. Whitehead's operation. 6. Injection of carbolic acid. 7. Clamp and cautery. 8. Ligature.

Of these it may be said that the first four methods enumerated are but very little used to-day. A man whose hobby is electricity will use electrolysis. Those who are rather timid will use the second and third method. The fourth method, which is not that of up-to-date surgery in the first place, has the very serious objection of liability of secondary hemorrhage. The fifth method gives an excellent result if successful, but unfortunately statistics show many failures. The sixth method is advocated by many physicians because it does not lay the patient up, but the course of treatment is long and tedious, extending over many weeks. This method consists of a 25 per cent. solution of carbolic acid in glycerin and water. Four minims are injected, with a hypodermic syringe, into the center of the pile, and may have to be repeated on several occasions. If care is not.used, sloughing and ulcers will result. Usually but little pain follows.

We now have left the two most popular methods, each of which has many advocates. Those in favor of clamp and cautery contend there is not the danger from hemorrhage, nor the after-pain; the operation can be performed with more dispatch than' by the ligature method. On the other hand, the advocates of the ligature method believe the simple ligature is easier handled than the cumbersome clamp. If prop. erly applied there is no danger of slipping and therefore no hemorrhage. The cautery needs the whole attention of one assistant and often will not work at the moment it is needed. The slough which follows 
is longer in healing than with the ligature method, where the mucous membrane may be partially drawn over the top of the stump with fine silk, leaving but little raw surface.

In their technic both operations resemble each other in a great many points. The patient having been properly prepared, and anesthetized and placed in the lithotomy position, the surgeon dilates the sphincter slowly but so completely that it will not regain its tone for two or three days. A soft sponge with string attached is then passed into the rectum above the site of operation to prevent descent of any fecal matter that preceding enemas may not have brought away. Now each pile is in turn seized with a tenaculum and pulled out from the wall to make it more prominent. The mucous membrance at its base is dissected away with curved scissors. If the clamp is the method preferred, it is now applied, the blade being transverse to the axis of the gut and fitted into the dissected surface at the base, then screwed up and that portion of the tumor outside of the clamp excised. The raw surface is seared with the cautery, at dull red heat, and the clamp slowly removed.

In the ligature method a double ligature of catgut is passed through the base of the pile and each half is tied separately; the pendent portion is then removed with scissors. Care must be taken here not to cut so close to the ligature that it will afterward slip. With fine silk the mucous membrane may now be drawn over the stump as much as possible. Each pile is taken in turn, whether by clamp or ligature, after which, the sponge having been removed, the surface is dusted with some of the numerous dusting powders; modoformol seems to have given the best results in my hands. An opium suppository now being inserted, a pyramid dressing of gauze is applied, held in place by a T-bandage. After-treatment consists of opiates in addition to the suppository, when necessary, for twelve hours. Catheterization is necessary as a rule. The bowels should be kept quiet for three days and an enema of olive-oil given preceding the movement.

149 Franklin St.

\section{FIFTY-ONE MASTOID CASES. *}

FIFTY OPERATIONS, INCLUDING FOUR BRAIN ABSCESSES AND ONE PERFORATION OF THE SIGMOID SINUS.

\section{BY D. MILTON GREENE, M.D.}

Eye, Ear, Nose and Throat Surgeon to U. B. A. Hospital and Holland Home for the Aged; Lecturer to U. B. A. Training School for Nurses.

GRAND RAPIDS, MICH.

For convenience and to prevent the necessity of giving in detail the histories of a long list of cases, which would add no interest to this report, I have divided the fifty-one mastoid operations into two classes. Class one includes those which presented nothing unusual for cases of suppurative mastoiditis. Class two includes all cases not included in class one.

In class one are thirty-six operations. Of these, twenty-four followed acute suppurative otitis media. Only twelve had chronic suppurative otitis. The operations were all performed with chisel and mallet. In six cases an opening was made from the antrum into the tympanum. In only five was there swelling over the mastoid process, two of which were in children aged eighteen months and two years respectively. All had a temperature ranging from 99 to $10 €$, and all recovered. In thirty-one the diagnosis was made from

\footnotetext{
* Read before the Western Ophthalmologic Association, Feb. 10, 1899.
}

the quantity and persistency of the aural discharge, temperature, chills, pain, and especially the swelling and protrusion at the upper and posterior wall of the tympanum and auditory canal. This class, therefore, presents nothing of special interest, except the fact that a diagnosis was made in each case, operation performed, and pus found in the cells and antra with the exception of three cases. In the cases where pus was not found, the cells and antra were in tensely congested, and I believe the operations were beneficial by their depleting effect on the parts, thereby cutting short the inflammatory process. No damage was done by the operation in any case.

In class two there are fifteen cases, each of which presents points of special interest. This includes one patient not operated on, who died, and autopsy was made confirming the diagnosis.

Case 37.-Mr. D., aged 48, gave a history of chronic aural discharge by spells for thirty years, from his left ear; swell. ing in temporal region for several weeks, extending mostly above and anterior to the ear; temperature 102 ; pulse 100 . There was evidence of pus formation, though the swelling was tense and no fluctuation. I incised the swelling and found a hole in the skull communicating with an extradural abscess located in the middle fossa. The abscess cavity was thoroughly curretted and drained and the case recovered.

Case 38.-Miss B. had chronic aural discharge which had resisted years of treatment. She took cold from a draught of air on the side of her affected ear, had chills, pain over mastoid and in ear, and temperature ranging from 99 to 102 ; no external swelling, edema nor redness over mastoid; tympanic walls at posterior superior portion swollen. The membranes of the auditory canal at the upper and back part were swollen and inflamed. Operation, on fifth day. revealed pus in the mastoid antrum, which was freely opened and scraped. A week later a retropharyngeal abscess formed, marked by a severe chill and temperature of 105 , and when opened discharged a half ounce of pue. The patient recovered in six weeks.

Case 39.-J. S., aged 38, had pain in the left temple extending from the ear forward over the face and brow. This continued for about three weeks. He had no discharge from the ear, but his wife said he had some several years before. He improved after the first week and went to work for several days, when he was again obliged to quit work and take to his bed. At the end of three weeks from the time he was taken, he began to be delirious by spells. When asked a question he would try to answer it, but would always say something other than an answer, as for instance: "Mary" (his wife's) or some other familiar name.

A brain specialist was called, and advised the family physician and family to call me to see if he might not have some ear trouble, which had given rise to the temporal pain. When I called I found him as stated above, but also with his left eye turned outward, pupils quite large though reacting to light, temperature 98, pulse 50. I could not get a good view with the ophthalmoscope, on account of his restlessness and struggles to get out of bed, but there was choked disc to some extent; bowels constipated. He had vomited some for three days whenever he drank water. He took no food, was quite emaciated but strong. Pus was seen behind the drumhead, which was slightly inflamed in Shrapnel's membrane, and there was a little offensive pus in the auditory canal. There was no looal swelling or signs of any kind over the mastoid, and no motor paralysis excepting of the internal rectus. He had difficulty in standing, with tendency to fall to the left. $\mathrm{He}$ was anxious and excited, grasping for the bed-clothes constantly and was determined to lie on his back or left side, constantly turning his face to the right as though hunting for something on that side.

I diagnosticated brain abscess. As the brain specialist believed no abscess existed and told the family so, stating that if $I$ thought there was I could oper. 